(2)

\section{OPEN ACCESS}

\title{
Tuberculosis in times of COVID-19
}

\author{
Alexandra Jaye Zimmer (D) ,'2 Joel Shyam Klinton (D) , ${ }^{2,3}$ Charity Oga-Omenka, 1,2 \\ Petra Heitkamp, ${ }^{2,3}$ Carol Nawina Nyirenda, ${ }^{4}$ Jennifer Furin, ${ }^{5}$ Madhukar Pai (D) ${ }^{1,2}$
}

\begin{abstract}
${ }^{1}$ Department of Epidemiology, Biostatistics and Occupational Health, McGill University, Montreal, Quebec, Canada ${ }^{2}$ McGill International TB Centre, Montreal, Quebec, Canada ${ }^{3}$ TB PPM Learning Network, Montreal, Quebec, Canada ${ }^{4}$ CITAMPlus, Lusaka, Zambia ${ }^{5}$ Department of Global Health and Social Medicine, Harvard Medical School, Boston, Massachusetts, USA
\end{abstract}

Correspondence to Madhukar Pai, Department of Epidemiology, Biostatistics and Occupational Health, McGill University, Montreal, QC H3A 1A2, Canada: madhukar.pai@mcgill.ca

Received 21 June 2021 Accepted 2 September 2021 Published Online First 17 September 2021

\section{Check for updates}

(c) Author(s) (or their employer(s)) 2022. Re-use permitted under CC BY. Published by BMJ.

\section{To cite: Zimmer AJ,} Klinton JS, Oga-Omenka C, et al. J Epidemiol Community Health 2022;76:310-316.

\section{ABSTRACT}

The COVID-19 pandemic has caused widespread disruptions to tuberculosis (TB) care and service delivery in 2020, setting back progress in the fight against TB by several years. As newer COVID-19 variants continue to devastate many low and middle-income countries in 2021, the extent of this setback is likely to increase. Despite these challenges, the TB community can draw on the comprehensive approaches used to manage COVID-19 to help restore progress and mitigate the impact of COVID-19 on TB. Our team developed the 'Swiss Cheese Model for Ending TB' to illustrate that it is only through multisectoral collaborations that address the personal, societal and health system layers of care that we will end TB. In this paper, we examine how COVID-19 has impacted the different layers of TB care presented in the model and explore how we can leverage some of the lessons and outcomes of the COVID-19 pandemic to strengthen the global TB response.

\section{INTRODUCTION}

Prior to COVID-19, tuberculosis (TB) was the leading infectious disease killer globally. In 2019 alone, there were approximately 10.0 million new TB infections and 1.5 million TB deaths globally. ${ }^{1}$ Over the past decades, efforts to curb the TB epidemic have gradually intensified. By the end of 2019, seventy-eight countries (including seven highburden countries (HBC)) were on track to achieve the 2020 End TB goals of reducing TB incidence by $80 \%$ and TB mortality by $90 \%$, compared with 2015 rates. $^{12}$ However, the COVID-19 pandemic and lockdown measures have created a massive global setback towards achieving these goals as TB services were disrupted at every level of the healthcare system.

\section{Impact of COVID-19 on TB services}

According to the Global Fund's snapshot report from 502 health facilities across Asia and Africa, TB referrals have fallen by 59\% in the second and third quarters of 2020 compared with the same period in 2019. ${ }^{3}$ Data from the World Health Organization (WHO) in March 2021 showed that COVID-19related disruptions have severely impacted over 84 countries with 1.4 million fewer people estimated to have received TB care in 2020, representing a $21 \%$ drop relative to 2019 (figure 1). ${ }^{4}$ In May 2020, the Stop TB Partnership published one of the earliest models on the impact of COVID-19 on TB, estimating a setback of at least 5-8 years in the fight against TB. ${ }^{5}$ More recently, a model by Hogan et al found that over the next 5 years TB deaths in high-burden settings could increase by up to $20 \% .^{6}$
These projections are a warning of what is to come unless a coordinated effort is implemented that addresses the layers of TB care.

During the second year of the pandemic, driven by newer, more transmissible variants (the Delta variant, in particular), the COVID-19 epicentres have shifted towards low- and middle-income countries, many of which are struggling to manage second, or even third, waves of COVID-19. South Asia and South America have been hit particularly hard in 2021. By May, Latin America and the Caribbean surpassed 1 million COVID-19 deaths, the majority of which occurred in Brazil. ${ }^{7}$ India's daily COVID-19 infections hit an all-time high with 414188 cases recorded on 6 May 2021 (which is likely an underestimation), with an estimated excess death toll of 3-5 million during the pandemic, ${ }^{8}$ while neighbouring Bangladesh, Nepal, Myanmar, Sri Lanka and Indonesia experienced similar surges. ${ }^{7}$ We are also starting to see worrying increases in COVID-19 cases across the African continent, with South Africa, Uganda, South Africa, the Democratic Republic of Congo, Namibia and Angola reporting new surges. ${ }^{9}$ Many of these are also TB HBCs, and now have the added burden of dealing with a 'syndemic' of TB and COVID-19. ${ }^{10}$

Because the devastation caused by the Delta variant in 2021 in HBCs was not accounted for in early 2020 projections and models, one may speculate that the impact of COVID-19 on TB disease and mortality rates may in fact be much worse than previously estimated. A recent review by McQuaid et al provides a detailed summary of studies that have quantified the country-level TB service disruptions among the $48 \mathrm{~TB}$, TB-HIV and multidrugresistant HBCs. ${ }^{11}$ The overall conclusion was that TB health services have decreased significantly in these settings due to COVID-19. In order to mitigate the impact of COVID-19 on TB, a comprehensive and coordinated approach to TB care and control that addresses the multiple layers of TB care is urgently needed.

\section{COVID-19 shows the need for a comprehensive approach for disease control}

Everyone is comfortable with the idea that COVID-19 requires multiple interventions, some personal (eg, masking, vaccinations), while others are societal (eg, social benefits, sick leave). The 'Swiss Cheese Respiratory Pandemic Defense' model created by Ian M Mackay outlines the multiple layers of these personal and shared responsibilities needed to prevent the spread of COVID-19. ${ }^{12}$

This model was adapted by our team to represent the TB context as the 'Swiss Cheese Model for 


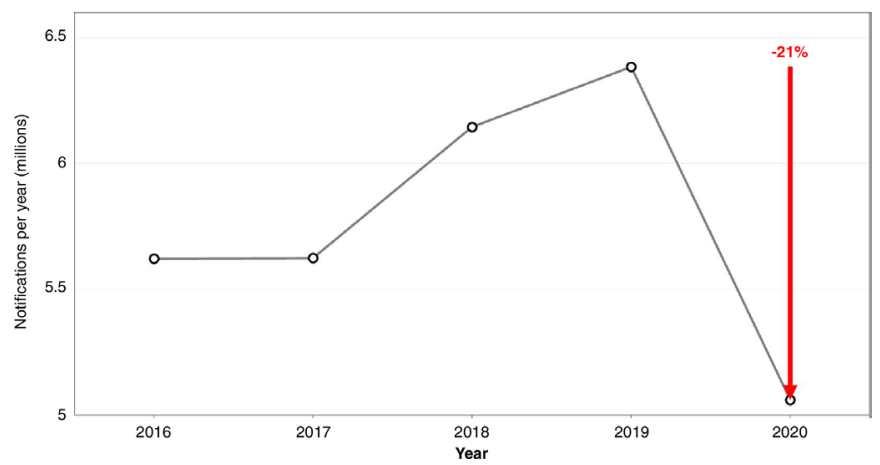

Figure 1 Annual change in tuberculosis (TB) case notification among 84 countries reporting monthly or quarterly TB case notification data (2016-2020). Arrow represents the per cent change in TB notifications relative to the previous year. Data from the $\mathrm{WHO}^{4}$

Ending TB', outlining the layers of interventions needed to end TB (figure 2). ${ }^{13}$ The TB Swiss Cheese Model has three broad levels: societal, personal and person-centric healthcare system. Within each level, layers of interventions have gaps ('holes') that lead to negative TB outcomes, and it is only by addressing these multiple layers that we protect individuals from either TB infection or TB-related mortality. Previously, TB care has primarily focused on empowering healthcare workers, emphasising vertical diagnostics and treatment systems. TB care also prioritised the 'simplistic' over the comprehensive, attempting to address only one or two aspects of the disease (eg, only diagnosing and treating people with a positive smear or people with drug-sensitive TB). Recently, more attention is being given towards strengthening person-centred care, as illustrated in the TB Swiss Cheese Model, and COVID-19 has further highlighted the importance of societal and personal factors. In this paper, we describe how COVID-19 has worn down some of the protective TB Swiss cheese layers, increasing the risk of poor outcomes for people with $\mathrm{TB}$, while simultaneously creating opportunities to reinforce other layers by leveraging some of the technology and public health tools used to combat COVID-19.

\section{SOCIETAL}

Tackle social determinants of health and inequities

The social determinants of health that lead to TB infection and mortality overlap with those related to COVID-19 infection and mortality. ${ }^{14}$ Socioeconomic status, lack of political will to spend on health, physical environment and demographic factors are strong predictors of adverse health outcomes for both diseases.

It is estimated that between 88 and 93 million individuals were pushed into extreme poverty in 2020 because of the pandemic, a number that is increasing during 2021. ${ }^{15}$ Food insecurity and hunger are also rising. As of April 2021, approximately 111 million additional people situated in 35 low and middle-income countries were living without sufficient food compared with April 2020. ${ }^{16}$ Poverty and poor nutritional status are inextricably linked with poor TB outcomes. ${ }^{17}$ In India, it is estimated that a population-level reduction in body mass index due to COVID-19-related disruptions to food access is expected to cause a $13.8 \%$ increase in TB incidence among the poor. ${ }^{18}$ Shelter-in-place orders have confined many vulnerable individuals and families to their homes, increasing the social challenges of economic and food insecurity while simultaneously increasing the rates of household TB transmission. ${ }^{19}$ As more individuals are pushed into poverty and hunger because of the pandemic, it is inevitable that more people will become infected with, and die of, TB.

While TB has many upstream determinants of health, it is also in itself a determinant of health for other diseases, including COVID-19. A study from Western Cape, South Africa, found that both current and prior TB significantly increased the risk of COVID-19 mortality. ${ }^{20}$ Another study from Khayelitsha, South Africa, found that a positive COVID-19 test was associated with a higher risk of mortality among people with rifampicinresistant (RR) TB (61\% mortality in RR-TB individuals with COVID-19). ${ }^{21}$

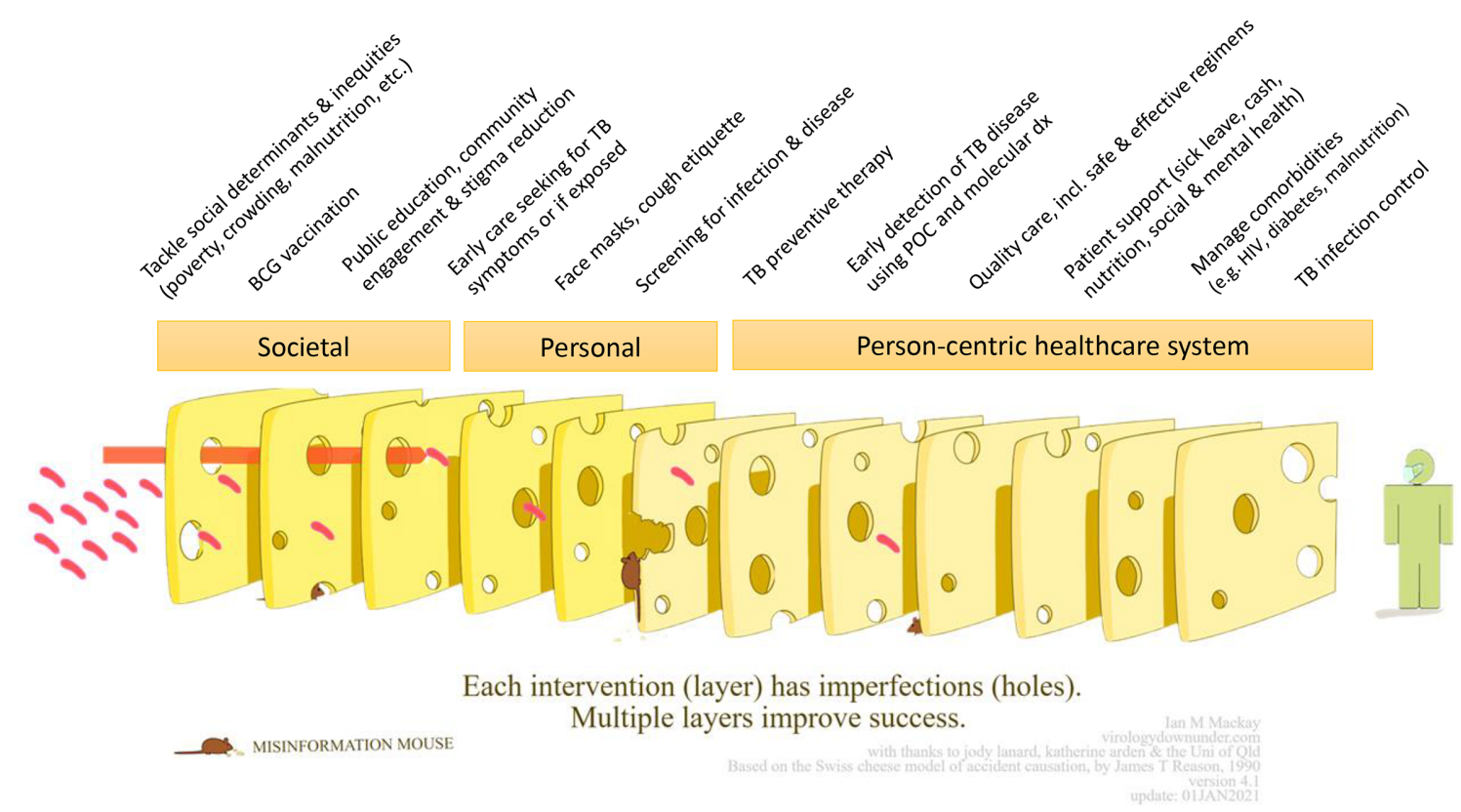

Figure 2 The Swiss Cheese Model for Ending TB (adapted for TB by Furin and Pai $\left[{ }^{13}\right]$, with permission from Dr lan Mackay). With input from Amrita Daftary, Petra Heitkamp, Joel Klinton, Emily MacLean, Lena Faust, Giorgia Sulis, Sophie Huddart and Anita Svadzian. POC, point of care; TB, tuberculosis. 
Providing TB care without addressing underlying comorbidities and social determinants of TB is inadequate, ${ }^{22}$ particularly in the wake of the COVID-19 pandemic. Further, addressing the social determinants of $\mathrm{TB}$ and reducing $\mathrm{TB}$ incidence directly reduces COVID-19 mortality. The pandemic has amplified the urgent need to develop effective interventions that address the health equity gap underlying barriers to universal health coverage in order to simultaneously control the spread and mitigate the impact of both COVID-19 and TB.

\section{Vaccines}

Vaccination is a key societal prevention strategy both for COVID-19 and TB. As of 1 June 2021, there were 287 candidate vaccines in development for COVID-19. Among these, 185 were in preclinical development and 102 in clinical development, 14 of which are approved. ${ }^{23}{ }^{24}$ Comparatively, there are only 18 TB candidate vaccines in development for adolescents and adults as of October 2020, none of which have moved beyond phase III of clinical trials. ${ }^{25}$ The only TB vaccine licensed for use is the BCG vaccine; a childhood vaccine developed in 1921 that offers variable protection with waning efficacy. ${ }^{26} 27$ The M72/ AS01E TB vaccine candidate is one of the most advanced TB vaccines for adults, having just completed phase IIb trials in early $2020,{ }^{28}$ and yet, the projected time to product availability is $2028 .^{29}$ This contrast is partly due to differences in political will and funding: between 2005 and 2019 TB vaccine research received US\$1.4 billion globally while the vaccine pillar of the Access to COVID-19 tools (ACT) Accelerator for COVID-19 received US\$9.5 billion globally within just over 1 year (as of 1 June 2021). ${ }^{30} 31$

While progress in vaccine development is typically hindered, the monumental advancements in COVID-19 vaccine development and technology, including the new benchmark set by the remarkable speed of development, present future opportunities for TB vaccine development. Additionally, there is hope that the technology behind the novel mRNA vaccines could be leveraged to develop an effective TB vaccine. ${ }^{32}$ Finally, the momentum behind COVID-19 vaccine development has given rise to promising collaborative initiatives for TB vaccine development, including the global road map for TB vaccine development launched by the European and Developing Countries Clinical Trials Partnership and the Amsterdam Institute for Global Health and Development. ${ }^{33}$

Until a more effective TB vaccine is available, it is essential that BCG vaccination levels are maintained. However, the United Nations estimates that 23 million children missed out on basic, routine childhood vaccines in $2020 .{ }^{34}$ Data from Pakistan and India also reveal sharp declines in the number of BCG vaccinations administered during the lockdown. ${ }^{35}$ Reasons for decreased coverage are linked to decreased access to health facilities due to lockdown measures as well as vaccine supply chain challenges. Maintaining supplies of BCG for infants during COVID-19 is essential to prevent severe forms of paediatric TB. ${ }^{37}$

\section{Public education, community engagement and stigma reduction}

Information, health education, communication, and addressing knowledge, attitudes and behaviours are essential disease prevention and control strategies. In TB control, these are often used in conjunction with active case finding and to promote treatment adherence. COVID-19 has brought many outreach campaigns and community drives to a halt. However, some programmes integrated TB and COVID-19 public awareness through public announcements, media campaigns and educational pamphlets that aimed to educate people about both diseases. ${ }^{38}$ For example, Mercy Corps in Pakistan helped disseminate information about TB and COVID-19 through mosque announcements and FHI 360 Philippines partnered with a media company to build social media campaigns. ${ }^{39}$ The adoption of such community-based educational models strengthens the person-centred approach to TB prevention. COVID-19 has also spearheaded the development of more advanced digital educational tools. For example, COVID-19 HealthAlert by Praekelt disseminates accurate and up-to-date COVID-19 information at a population level via WhatsApp, and was able to reach more than 6 million individuals within the first 7 weeks of deployment. ${ }^{40}$

Nevertheless, the rapid dissemination of misinformation on digital communication platforms and social media can be a threat to public health. For TB control, misinformation can result in delayed care seeking, increased transmission and poor health outcomes. ${ }^{41}$ Because of the overlapping symptoms between COVID-19 and TB, stigma and misinformation on TB have increased significantly. Results from a global communitybased survey found that $56 \%$ of people with TB from Kenya $(n=159)$ reported feelings of shame because of the overlapping symptoms with COVID- $19 .{ }^{42}$ In order to minimise TB-related stigma and ensure individuals make informed health decisions, it is essential that information on TB continues to be delivered to at-risk communities.

Another important aspect of community engagement to consider is contact tracing. Contact tracing is a core strategy used to curb both TB and COVID-19 transmission, particularly in regions where the COVID-19 epidemic curve is flattened or declining. Effective contact tracing may help address stigma, community trust and engagement, and use of operational guidelines for both TB and COVID-19. ${ }^{43}$ Many of the COVID-19 contact tracing initiatives have drawn on TB programme staff expertise, though sometimes at the detriment to TB prevention and control efforts. A report published by the Centers for Disease Control and Prevention's (CDC) Division of Tuberculosis Elimination found that among $50 \mathrm{~TB}$ programmes in the USA, $60 \%$ experienced a partial or high impact on TB staffing capacity for outreach (including contact investigations). ${ }^{44}$ Contact tracing initiatives are some of the more labour and resource-intensive strategies for both TB and COVID-19. Unless proper resource allocation guidelines are in place that simultaneously address the needs of both TB and COVID-19, one disease will become deprioritised, as is currently the case for TB. In order to address the growing backlog of undiagnosed TB cases, an integrated, dual TB and COVID-19 contact tracing approach is needed to maximise the use of staff and resources. ${ }^{45}$

\section{PERSONAL}

\section{Early care seeking for TB symptoms or if exposed}

During the first months of the pandemic, TB notifications decreased sharply in HBCs. Restriction in movement, fear of contracting or being misdiagnosed with COVID-19, and stigma all impacted care seeking. The global survey found that $50 \%$ of people with TB in Kenya $(n=159)$ reported having trouble accessing TB care due to restricted access to public transportation (cost and availability of transport). ${ }^{42}$ From the same survey, $62 \%$ of people with TB from India $(n=58)$ reported that they did not want to visit their regular facility out of fear of contracting COVID-19. One study from an infectious disease referral hospital in Italy found that between March and August 
2020, the median delay to seeking a TB diagnosis increased by 30 days compared with the same period in $2019 .{ }^{46}$ This delay in care seeking has contributed to a growing pool of missed people, leading to surges in community-based TB transmission, more severe forms of $\mathrm{TB}$, and $\mathrm{TB}$ mortality. ${ }^{47}$

\section{Personal infection control practices, such as face masks}

COVID-19 forced governments to impose respiratory infectious disease prevention and control measures on individuals within public spaces. Given the overlap in aerosol disease transmission dynamics between COVID-19 and TB, many of these measures also apply to TB prevention and control. At the individual level, masks have been central to the COVID-19 response. ${ }^{48}$ The use of masks by people affected by TB or by people who interact with people who have TB has historically been met with limited compliance due to 'stigma, restricted access, discomfort, and perceived liberty deprivation'. ${ }^{49}$ One pre-COVID-19 study in Uganda found that wearing a mask was 'embarrassing' for persons with $\mathrm{TB}$ as it emphasised their infectiousness while another pre-COVID-19 study in South Africa found that only $2 \%$ of participants wore a face mask (which increased to $17 \%$ after an education and awareness-raising intervention). ${ }^{50}{ }^{51}$ As noted by Driessche et al, the near-universal enforcement of masks and other infection prevention and control (IPC) measures may help normalise the use of such measures against airborne infectious diseases, including $\mathrm{TB}$, and reduce stigma. ${ }^{49}$ When the pandemic recedes and the enforcement of masks and other infection control practices becomes less stringent, it is important that behaviours that promote improved infection control and destigmatise mask use are maintained in the context of TB care.

\section{Screening for infection and disease}

Many community-based screening, contact tracing, and active case finding initiatives were hindered during COVID-19, resulting in a reduction or discontinuation of TB screening efforts. ${ }^{52}$ In Nigeria, the Wellness on Wheels campaign, which uses mobile diagnostic units to screen patients with digital X-ray machines, experienced a $67 \%$ decrease in patients screened between 8 and 29 March $2020 .{ }^{39}$ A rapid assessment of intermediary agencies involved in TB care also reported screening and outreach disruptions across seven HBCs. ${ }^{39}$

Shelter-at-home orders have made screening more challenging as individuals are not able to access health facilities. Also, people with TB who are confined to their homes increase the risk of household transmission, particularly among vulnerable populations living in crowded households. ${ }^{53}$ As community and household-based transmission increases, it is essential to promote community screening initiatives that follow person-centred models. The 'Tuberculosis Neighborhood Expanded Testing' Project in South Africa is an example of a successful communitybased screening model which led to good linkage to care during COVID-19. This 'door-to-door' approach of collecting sputum samples enabled $92 \%$ of individuals that provided a sample to be tested for TB. ${ }^{54}$

Because COVID-19 and TB share similar symptoms, community outreach and screening activities for both diseases need to be integrated. ${ }^{556}$ While such bidirectional screening activities have already been applied in many countries burdened by both diseases, including India and Nigeria, the extent of the scale-up of such initiatives is unknown. ${ }^{5758}$

The rapid innovation in tools driven by the COVID-19 pandemic may present opportunities for improved communitybased TB screening, including testing closer to homes. Such innovations include over-the-counter antigen detection tests, ${ }^{59}$ face masks that detect SARS-CoV-2 RNA in aerosols ${ }^{60}$ and mouth/saliva swabs for facilitated sample collection. ${ }^{61}$ Innovators must work towards integrating these new tools for improved TB screening and care. ${ }^{40}$

\section{PERSON-CENTRIC HEALTHCARE SYSTEM \\ TB preventive therapy}

As many individuals with TB are confined at home, it is essential that household contacts receive effective preventive therapies. According to McQuaid et al, Brazil, Kenya, the Philippines, Russia, South Africa, Sierra Leone and Zambia reported a $30 \%-70 \%$ decrease in preventive therapy enrolment. ${ }^{11}$ One model published in November 2020 estimated that the proportion of people living with HIV receiving isoniazid preventive therapy (IPT) in Kenya could drop to $10 \%$ due to disruptions caused by COVID-19 lockdowns. ${ }^{62}$

Despite this, COVID-19 has successfully altered stigmatised perceptions surrounding the administration of anti-TB preventive therapy in some settings. A programme in Khayelitsha, South Africa that provides TB preventive therapy to children and adolescents exposed to RR-TB in households found that 'there is increased acceptance of tuberculosis preventive therapy compared with before the pandemic, possibly due to changed risk perceptions'. ${ }^{19}$ Thus, while the pandemic may currently affect the ability of health providers to administer IPT, changes in risk perception brought about by the pandemic may help improve IPT uptake in the long term.

\section{Early detection of TB disease using point-of-care and molecular diagnostics}

From a health systems perspective, the lack of diagnostic capacity to support simultaneous testing for both COVID-19 and TB presented a major barrier to adequate $\mathrm{TB}$ control measures. With the release of the GeneXpert Xpress SARS-CoV-2 cartridge by Cepheid, global networks of GeneXpert machines normally used to test TB were disrupted. Forty-three countries, including 13 HBCs, reported using GeneXpert for COVID-19 testing instead of TB testing.

Instead of reallocating diagnostic resources from $\mathrm{TB}$ towards COVID-19, the WHO recommends multidisease testing strategies, as is already being implemented in India. ${ }^{63}$ The overlapping epidemiology and clinical characteristics of these diseases is a call for healthcare providers and researchers to move away from vertical disease-specific approaches, which has long impeded TB control, towards comprehensive and integrated methods. ${ }^{64}$ Similar to screening tools, COVID-19 has spearheaded the development of novel diagnostics. Promising tools include point-of-care chest radiology and cough detection technologies that can potentially be used to differentiate COVID-19 from TB and other respiratory ailments in low-resource settings. ${ }^{40}$

\section{Quality of care, including safe and effective regimens}

Even before COVID-19, TB quality of care was considered suboptimal; however, the pandemic has only worsened the quality of care people with TB receive. ${ }^{65}$ Failure to provide treatment in a timely fashion is a TB-related human rights violation. ${ }^{66}$ Therefore, the appropriateness of rigid adherence support strategies, such as facility-based directly observed therapy (DOT), has come under increased scrutiny during the pandemic. ${ }^{67}$ In many settings, facility-based DOT became impossible during COVID-19-patients could not access the health centre or were not allowed in, resulting in many people missing therapy. ${ }^{67} \mathrm{To}$ 
overcome the barriers created by facility-based DOT, there has been a shift towards strengthening community-based TB treatment services to ensure patients get the medicine in a timely fashion. ${ }^{68}$ Other strategies include multimonth dispensing and home delivery of treatment. ${ }^{67}$ In the Philippines, the Department of Health issued a memorandum urging TB services to provide a 1-month supply of anti-TB medication instead of going to the facility for their daily dose. ${ }^{69}$ This was issued after noting a near 10 -fold decrease in patients receiving their treatment during the first quarter of 2020 compared with the same time period in 2019. While home-based options, including delivery of medications to patients' homes, have made treatment more accessible, counselling and treatment literacy support were often missing. ${ }^{7071}$ The increased acceptance and uptake of video DOT (vDOT) and other telemedicine options has helped overcome the barriers to non-medical support systems; however, this may only be an option in locations that have sufficient data/internet coverage. ${ }^{67}$ Therefore, community-based care services must remain available in addition to vDOT strategies in order to provide more flexible treatment options during and beyond the COVID-19 pandemic.

\section{Patient support and managing comorbidities}

While anti-TB therapy is needed to manage disease progression, it is not sufficient to prevent mortality. Non-medical support that places the person at the centre of care is essential for successful outcomes. This includes nutritional, financial, counselling, and psychosocial support. ${ }^{72}$ As previously discussed, many of these support systems fell apart during COVID-19. It is important for health systems to adapt their health delivery strategies to include non-medical support, particularly for vulnerable populations burdened by social determinants and comorbidities that increases their risk of TB and/or COVID-19. A recent survey, which collected responses from 567 people with TB across 64 countries, found that $31 \%$ of respondents reported that nonmedical support 'was much harder or impossible to access' during COVID-19. ${ }^{73}$

It is also important to recognise that not all people with $\mathrm{TB}$ require the same amount of care, as individuals with multidrug resistance or other comorbidities may require additional support. Comorbidities such as HIV and diabetes are particularly important to address as they increase the chance of mortality among people with TB. A 2020 WHO rapid assessment of COVID-19 on non-communicable diseases found that $49 \%$ of the 163 ministries surveyed reported partial or complete disruption to services for diabetes and diabetes-related complications. ${ }^{74}$ COVID-19 also disrupted HIV services, including screening and antiretroviral therapy delivery. ${ }^{75}$ These disruptions have made comprehensive care even more inaccessible for people with TB living with comorbidities. That said, the immense efforts that have gone into characterising COVID-19 mortality risk factors have highlighted the importance of managing comorbidities, such as diabetes and HIV, to reduce deaths. ${ }^{767}$ In order to make progress towards achieving TB elimination goals, we must work towards integrated TB care programmes that address the needs of individuals living with comorbidities, including options that integrate remote and home-based care.

\section{TB infection control}

COVID-19 has promoted capacity-building efforts for IPC within healthcare systems (eg, use of masks and personal protective equipment (PPE)). Historically, adherence to IPC guidelines for respiratory diseases has been poor. ${ }^{78}$ As IPC guidelines for
COVID-19 infection control are reinforced and adopted, this will facilitate the control and prevention of $\mathrm{TB}$ within healthcare facilities.

This awareness and capacity building is particularly important for primary care facilities, which play an important role for early TB detection and care, both before and during COVID-19. In Pakistan, many tertiary and secondary care hospitals closed their outpatient facilities to minimise COVID-19 transmission. ${ }^{79}$ As a result, many patients with $\mathrm{TB}$ were redirected to primary healthcare centres. An assessment of these primary facilities revealed low adherence to IPC measures in addition to poor supply chain management of drugs and consumables. In response, Pakistan's National TB Program trained healthcare professionals at primary facilities on proper use of PPE, supplemented by 'comprehensive educational modules on infection control which were prepared and delivered through online training to field staff' ${ }^{79}$ Thus, while COVID-19 has put enormous strains on healthcare systems globally, it also raised awareness of important infectious respiratory disease IPC measures in healthcare settings. Greater use of masks by patients, PPE by providers, and engineering controls such as ultraviolet light fixtures and better ventilation will greatly help nosocomial transmission of both COVID-19 and TB.

\section{CONCLUSION}

The severe disruptions of $\mathrm{TB}$ care and services caused by COVID-19 have set back progress towards achieving global TB targets, including the 2018-2022 United Nations HighLevel Meeting targets on TB treatment and prevention as well as the End TB objective of ending the TB epidemic by $2035 .^{80}$ In order to restore progress towards $\mathrm{TB}$ elimination, a more refined and multifaceted approach to managing TB is needed. The Swiss Cheese Model for Ending TB highlights that single layers of protection are insufficient to end TB, and instead stresses the need for a holistic approach to TB care that calls for the strengthening of societal, personal and health system layers simultaneously. While these layers are essential to TB control, other cross-cutting and programmatic considerations must be re-evaluated in order to adapt to the current global situation under COVID-19. Such actions include reaffirming political support, scaling up TB investments and improving the timeliness and quality of TB data reporting. There is no magic bullet to managing TB. Public health responses are not single interventions but closely intertwined with societal beliefs and actions.

Correction notice This article has been corrected since it first published. Figure 1 formatting has been corrected and reference 2 has been corrected.

Twitter Alexandra Jaye Zimmer @alexjzimmer, Joel Shyam Klinton@drjoelklinton, Petra Heitkamp @PetraHeitkamp and Madhukar Pai @paimadhu

Acknowledgements The authors used publicly available data (already reported in the media) for displaying the impact of COVID-19 on tuberculosis services. We thank Amrita Daftary, Emily MacLean, Lena Faust, Giorgia Sulis, Sophie Huddart and Anita Svadzian for their input on the 'Swiss Cheese Model for Ending TB'.

Contributors JSSK, PH, JF and MP developed the Swiss Cheese Model for Ending TB used to structure this manuscript. AJZ, JSSK, CO-O, PH and MP conceived the manuscript. AJZ wrote the original manuscript with input from all authors.

Funding This work was supported, in whole or in part, by the Bill \& Melinda Gates Foundation.

Competing interests MP serves as an advisor to the Bill \& Melinda Gates Foundation, WHO Stop TB Partnership and Foundation for Innovative New Diagnostics.

Patient consent for publication Not required.

Provenance and peer review Commissioned; externally peer reviewed.

Data availability statement No data are available. Data sharing is not applicable to this article as no new data were created or analysed in this study. 
Open access This is an open access article distributed in accordance with the Creative Commons Attribution 4.0 Unported (CC BY 4.0) license, which permits others to copy, redistribute, remix, transform and build upon this work for any purpose, provided the original work is properly cited, a link to the licence is given, and indication of whether changes were made. See: https://creativecommons.org/ licenses/by/4.0/

\section{ORCID iDs}

Alexandra Jaye Zimmer http://orcid.org/0000-0002-6047-0923

Joel Shyam Klinton http://orcid.org/0000-0003-4850-3961

Madhukar Pai http://orcid.org/0000-0003-3667-4536

\section{REFERENCES}

1 World Health Organization. Global tuberculosis report 2020, 2020. Available: https:// www.who.int/publications//item/9789240013131 [Accessed 15 Aug 2021].

2 World Health Organization. The end TB strategy, 2015. Available: https://www.who. int/tb/End_TB_brochure.pdf [Accessed 10 Mar 2021].

3 The Global Fund. The Impact of COVID-19 on HIV, TB and Malaria Services and Systems for Health - Updates - The Global Fund to Fight AIDS, Tuberculosis and Malaria, 2021. Available: https://www.theglobalfund.org/en/updates/other-updates/ 2021-04-13-the-impact-of-covid-19-on-hiv-tb-and-malaria-services-and-systems-forhealth/ [Accessed 25 May 2021]

4 World Health Organization. Impact of the COVID-19 pandemic on TB detection and mortality in 2020, 2021. Available: https://www.who.int/publications/m/item/impactof-the-covid-19-pandemic-on-tb-detection-and-mortality-in-2020 [Accessed 21 May 2021].

5 Stop TB Partnership. The devastating effect of the COVID-19 pandemic on the TB Response-A minimum of 5 years of progress lost and 6 million additional people ill with $T B, 2020$

6 Hogan AB, Jewell BL, Sherrard-Smith E, et al. Potential impact of the COVID-19 pandemic on HIV, tuberculosis, and malaria in low-income and middle-income countries: a modelling study. Lancet Glob Health 2020;8:e1132-41.

7 Dong E, Du H, Gardner L. An interactive web-based dashboard to track COVID-19 in real time. Lancet Infect Dis 2020;20:533-4.

8 Anand A, Sandefur J, Subramanian A. Three New Estimates of India's All-Cause Excess Mortality during the COVID-19 Pandemic. Cent Glob Dev Work Pap 2021;416 www. cgdev.org

9 Mwai P. Coronavirus in Africa: concern growing over third wave of Covid-19 infections. BBC News, 2021. https://www.bbc.com/news/world-africa-53181555

10 Pai M. Tuberculosis and Covid-19: fighting a deadly Syndemic. Forbes, 2020. Available: https://www.forbes.com/sites/madhukarpai/2020/09/26/tuberculosis-and-covid-19fighting-a-deadly-syndemic/?sh=36fcac4b24c5 [Accessed 02 Jun 2021].

11 McQuaid CF, Vassall A, Cohen T, et al. The impact of COVID-19 on TB: a review of the data. Int J Tuberc Lung Dis 2021;25:436-46.

12 Roberts S. The Swiss Cheese Model of Pandemic Defense - The New York Times, 2020. Available: https://www.nytimes.com/2020/12/05/health/coronavirus-swiss-cheeseinfection-mackay.html [Accessed 25 May 2021].

13 Furin J, Pai M. We went all-out to tackle Covid-19 - TB needs the same approach. The telegraph, 2020. Available: https://www.telegraph.co.uk/global-health/science-anddisease/went-all-out-tackle-covid-19-tb-needs-approach/ [Accessed 19 Apr 2021].

14 Duarte R, Aguiar A, Pinto M, et al. Different disease, same challenges: social determinants of tuberculosis and COVID-19. Pulmonology 2021;27:338-44.

15 et alLakner C, Yonzan N, Mahler D. Updated estimates of the impact of COVID-19 on global poverty: looking back at 2020 and the outlook for 2021. world bank blogs, 2021. Available: https://blogs. worldbank.org/opendata/updated-estimates-impactcovid-19-global-poverty-looking-back-2020-and-outlook-2021 [Accessed 28 May 2021].

16 The World Bank. Food security and COVID-19, 2021. Available: https://www. worldbank.org/en/topic/agriculture/brief/food-security-and-covid-19 [Accessed 28 May 2021].

17 Lönnroth K, Williams BG, Cegielski P, et al. A consistent log-linear relationship between tuberculosis incidence and body mass index. Int J Epidemiol 2010;39:149-55.

18 Bhargava A, Bhargava M, Juneja A. Social determinants of tuberculosis: context, framework, and the way forward to ending TB in India. Expert Rev Respir Med 2021;15:867-83.

19 Mohr-Holland E, Douglas-Jones B, Apolisi I, et al. Tuberculosis preventive therapy for children and adolescents: an emergency response to the COVID-19 pandemic. Lancet Child Adolesc Heal 2021;5:159-61.

20 Boulle A, Davies M-A, Hussey H. Risk factors for coronavirus disease 2019 (COVID-19) death in a population cohort study from the Western Cape Province, South Africa. Clin Infect Dis 2020;14:16.

21 Mohr-Holland E, Daniels J, Douglas-Jones B, et al. A positive COVID-19 test is associated with high mortality in RR-TB-HIV patients. Int I Tuberc Lung Dis 2021;25:409-12.

22 Lönnroth K, Jaramillo E, Williams BG. Tuberculosis: the role of risk factors and social determinants. Equity, Soc Determ public Heal Program 2010;219 https://www.
researchgate.net/publication/301608294_Tuberculosis_the_role_of_risk_factors_ and_social_determinants

23 World Health Organization. Draft landscape and tracker of COVID-19 candidate vaccines, 2021. Available: https://www.who.int/publications/m/item/draft-landscapeof-covid-19-candidate-vaccines [Accessed $10 \mathrm{Mar}$ 2021].

24 Faust L, Raithby L. The 100th anniversary of a vaccine against a deadly disease: not a cause for celebration | health affairs. Health Affairs, 2021. https://www. healthaffairs. org/do/

25 TuBerculosis Vaccine Initiative (TBVI). Pipeline of vaccines, 2020. Available: https:// www.tbvi.eu/what-we-do/pipeline-of-vaccines/ [Accessed 03 Mar 2021].

26 Whittaker E, Nicol MP, Zar HJ, et al. Age-related waning of immune responses to BCG in healthy children supports the need for a booster dose of BCG in TB endemic countries. Sci Rep 2018;8:15309.

27 Romano M, Huygen K. An update on vaccines for tuberculosis - there is more to it than just waning of BCG efficacy with time. Expert Opin Biol Ther 2012;12:1601-10.

28 Tait DR, Hatherill M, Van Der Meeren 0, et al. Final Analysis of a Trial of M72/ AS01 Vaccine to Prevent Tuberculosis. N Engl J Med 2019;381:2429-39.

29 World Health Organization. M72/ASO1E Tuberculosis Vaccine Candidate - ConsensusGenerating Consultation on the Development Pathway, 2019.

30 Treatment Action Group. Tuberculosis research funding trends, 2005-2019, 2020. Available: https://www.treatmentactiongroup.org/wp-content/uploads/2020/12/tbrd_ 2020_final_web.pdf [Accessed 03 Mar 2021].

31 World Health Organization. Access to COVID-19 tools funding commitment tracker, 2021. Available: https://www.who.int/publications/m/item/access-to-covid-19-toolstracker [Accessed 03 Mar 2021].

32 Merelli A. What does the Covid-19 vaccine mean for HIV, TB, and the flu? - quartz. quartz, 2020. Available: https://qz.com/1945114/what-does-the-covid-19-vaccinemean-for-hiv-tb-and-the-flu/ [Accessed 02 Jun 2021].

33 EDCTP. EDCTP and AIGHD launched a global roadmap for tuberculosis vaccine development - EDCTP. Available: https://www.edctp.org/news/edctp-and-aighdlaunched-a-global-roadmap-for-tuberculosis-vaccine-development/\# [Accessed 17 May 2021].

34 World Health Organization. COVID-19 pandemic leads to major backsliding on childhood vaccinations, new who, UNICEF data shows, 2021. Available: https://www. who.int/news/item/15-07-2021-covid-19-pandemic-leads-to-major-backsliding-onchildhood-vaccinations-new-who-unicef-data-shows [Accessed 20 Aug 2021].

35 Chandir S, Siddiqi DA, Mehmood M, et al. Impact of COVID-19 pandemic response on uptake of routine immunizations in Sindh, Pakistan: an analysis of provincial electronic immunization registry data. Vaccine 2020;38:7146-55.

36 Shrinivasan R, Rane S, Pai M. India's syndemic of tuberculosis and COVID-19. BMJ Glob Heal 2020;5:3979. doi:10.1136/bmjgh-2020-003979

37 Namkoong H, Horita N, Ebina-Shibuya R. Concern over a COVID-19-related BCG shortage. Int J Tuberc Lung Dis 2020;24:642-3.

38 Mugoni PC. Lessons for the COVID-19 response from a multidrugresistant tuberculosis study in South Africa: how public health promotion can reach vulnerable young women. S Afr J Public Health 2020;4:34. doi:10.7196/SHS.2020.v4i2.117

39 Klinton JS, Heitkamp P, Rashid A. One year of COVID-19 and its impact on private provider engagement for TB - A Rapid Assessment of Intermediary NGOs in seven High TB burden countries 2021. (In press).

40 Ruhwald M, Carmona S, Pai M. Learning from COVID-19 to reimagine tuberculosis diagnosis. Lancet Microbe 2021;2:e169-70.

41 Cremers AL, de Laat MM, Kapata N, et al. Assessing the consequences of stigma for tuberculosis patients in urban Zambia. PLoS One 2015;10:e0119861.

42 Action global health advocacy partnership, global coalition of TB activists, global TB caucus, et al. The impact of COVID-19 on the TB epidemic: a community perspective, 2020. Available: https://spark.adobe.com/page/xJ7pygvhrlAqW/ [Accessed 10 Mar 2021].

43 World Health Organization. Operational guide for engaging communities in contact tracing, 2021. Available: https://www.who.int/publications/i/item/WHO-2019-nCoVContact_tracing-Community_engagement-2021.1-eng [Accessed 16 Aug 2021].

44 Cronin AM, Railey S, Fortune D, et al. Notes from the Field: Effects of the COVID-19 Response on Tuberculosis Prevention and Control Efforts - United States, March-April 2020. MMWR Morb Mortal Wkly Rep 2020;69:971-2.

45 USAID, Stop TB Partnership. COVID-19 contact tracing and TB contact investigation: an integrated implementation approach, 2021. Available: http://stoptb.org/assets/ documents/covid/COVID-TB Integrated Contact Investigation_March2021.pdf [Accessed 16 Aug 2021].

46 Di Gennaro F, Gualano G, Timelli L, et al. Increase in tuberculosis diagnostic delay during first wave of the COVID-19 pandemic: data from an Italian infectious disease referral hospital. Antibiotics 2021;10:272-10.

47 Malik AA, Safdar N, Chandir S, et al. Tuberculosis control and care in the era of COVID-19. Health Policy Plan 2020;35:1130-2.

48 Chu DK, Akl EA, Duda S, et al. Physical distancing, face masks, and eye protection to prevent person-to-person transmission of SARS-CoV-2 and COVID-19: a systematic review and meta-analysis. Lancet 2020;395:1973-87.

49 Driessche KV, Mahlobo PZ, Venter R. Face masks in the post-COVID-19 era: a silver lining for the damaged tuberculosis public health response? 2021;9. doi:10.1016/ S2213-2600(21)00020-5 
50 Buregyeya E, Mitchell EMH, Rutebemberwa E, et al. Acceptability of masking and patient separation to control nosocomial tuberculosis in Uganda: a qualitative study. $J$ Public Health 2012;20:599-606. doi:10.1007/s10389-012-0503-1

51 Haeusler IL, Knights F, George V, et al. Improving TB infection control in a regional hospital in the Eastern Cape, South Africa. BMJ Open Qual 2019;8:bmjoq2018-000347. doi:10.1136/bmjoq-2018-000347

52 Odume B, Falokun V, Chukwuogo O, et al. Impact of COVID-19 on TB active case finding in Nigeria. Public Health Action 2020;10:157-62. doi:10.5588/pha.20.0037

53 Francisco NM, Fang Y-M, Ding L, et al. Diagnostic accuracy of a selected signature gene set that discriminates active pulmonary tuberculosis and other pulmonary diseases. J Infect 2017;75:499-510.

54 Zokufa N, Lebelo K, Hacking D, et al. Community-Based TB testing as an essential part of TB recovery plans in the COVID-19 era. Int J Tuberc Lung Dis 2021;25:406-8. doi:10.5588/ijtld.21.0077

55 Padma TV. India looks to Al to tackle TB, COVID-19. BioWorld, 2021. Available: https://www.bioworld.com/articles/502207-india-looks-to-ai-to-tackle-tb-covid-19 [Accessed 10 Mar 2021].

56 Kozlov M. Al-Assisted cough tracking could help detect the next pandemic. The Scientist 2020 https://www.the-scientist.com/news-opinion/ai-assisted-coughtracking-could-help-detect-the-next-pandemic-68233

57 Ministry of Health and Family welfare of India (MOHFW). Guidance note on bidirectional TB-COVID screening and screening of TB among ILI/SARI cases continue treatment for TB, 2020. Available: https://www.mohfw.gov.in/pdf/1TBCOVIDscreenin gguidancenote.pdf [Accessed 19 May 2021].

58 World Health Organization. Nigeria: integrating COVID-19 screening into TB surveillance activities, 2020. Available: https://www.who.int/news-room/featurestories/detail/nigeria-integrating-covid-19-screening-into-tb-surveillance-activities [Accessed 19 May 2021].

59 The global fund. COVID-19 response: Antigen-Detection diagnostic test procurement, 2020. Available: https://www.theglobalfund.org/en/sourcing-management/updates/ 2020-09-15-covid-19-response-antigen-detection-diagnostic-test-procurement/ [Accessed 10 Jun 2021].

60 Oliver S. High-Tech face masks aim to step up the fight against Covid-19. Wall Street Journal, 2021. Available: https://www.wsj.com/articles/high-tech-face-masks-aim-tostep-up-the-fight-against-covid-19-11616846402 [Accessed 10 Jun 2021].

61 Teo AKJ, Choudhury Y, Tan IB, et al. Saliva is more sensitive than nasopharyngeal or nasal swabs for diagnosis of asymptomatic and mild COVID-19 infection. Sci Rep 2021;11:3134.

62 Cilloni L, Fu H, Vesga JF, et al. The potential impact of the COVID-19 pandemic on the tuberculosis epidemic a modelling analysis. EClinica/Medicine 2020;28:100603.

63 The Global Fund. COVID-19 Situation Report \#41, 2021. Available: https://www. theglobalfund.org/en/covid-19/ [Accessed 17 May 2021].

64 Raviglione MC, Pio A. Evolution of WHO policies for tuberculosis control, 1948-2001. Lancet 2002:359:775-80

65 Pai M, Temesgen Z. Quality of tuberculosis care, 2021. Available: https://www.mcgill. ca/tb/files/tb/quality_tb_care_ebook.pdf [Accessed 19 May 2021].
66 Stop TB Partnership. Declaration of the rights of people affected by tuberculosis, 2019.

67 Zimmer AJ, Heitkamp P, Malar J, et al. Facility-based directly observed therapy (dot) for tuberculosis during COVID-19: a community perspective. I Clin Tuberc Other Mycobact Dis 2021;24:100248.

68 Chiang C-Y, Islam T, Xu C, et al. The impact of COVID-19 and the restoration of tuberculosis services in the Western Pacific region. Eur Respir J 2020;56. doi:10.1183/13993003.03054-2020. [Epub ahead of print: 22 Oct 2020].

69 Republic of Philippines Department of Health. DOH urges TB patients to get 1-MONTH supply of MEDS during COVID-19 quarantine press Release/24 March 2020, 2020. Available: https://doh.gov.ph/doh-press-release/-DOH-URGES-TBPATIENTS-TO-GET-1-MONTH-SUPPLY-OF-MEDS-DURING -COVID-19-QUARANTINE [Accessed 10 Mar 2021].

70 Keene C, Mohr-Holland E, Cassidy T, et al. How COVID-19 could benefit tuberculosis and HIV services in South Africa. Lancet Respir Med 2020;8:844-6. doi:10.1016/ S2213-2600(20)30311-8

71 Klinton JS, Oga-Omenka C, Heitkamp P. TB and COVID - Public and private health sectors adapt to a new reality. J Clin Tuberc Other Mycobact Dis 2020;21:100199.

72 Bhargava A, Bhargava M. Tuberculosis deaths are predictable and preventable: comprehensive assessment and clinical care is the key. J Clin Tuberc Other Mycobact Dis 2020;19:100155

73 Khan MS, Rego S, Rajal JB, et al. Mitigating the impact of COVID-19 on tuberculosis and HIV services: a cross-sectional survey of 669 health professionals in 64 low and middle-income countries. PLoS One 2021;16:e244936.

74 World Health Organization. COVID-19 significantly impacts health services for noncommunicable diseases, 2021. Available: https://www.who.int/news/item/01-062020-covid-19-significantly-impacts-health-services-for-noncommunicable-diseases [Accessed 03 Jun 2021].

75 Dorward J, Khubone T, Gate K, et al. The impact of the COVID-19 lockdown on HIV care in 65 South African primary care clinics: an interrupted time series analysis. Lancet HIV 2021;8:e158-65.

76 Abdi A, Jalilian M, Sarbarzeh PA, et al. Diabetes and COVID-19: a systematic review on the current evidences. Diabetes Res Clin Pract 2020;166:10.1016/j. diabres.2020.108347.

77 Ssentongo P, Heilbrunn ES, Ssentongo AE, et al. Epidemiology and outcomes of COVID-19 in HIV-infected individuals: a systematic review and meta-analysis. SC Rep 2021;11:10.1038/s41598-021-85359-3.

78 Houghton C, Meskell P, Delaney H. Barriers and facilitators to healthcare workers' adherence with infection prevention and control (IPC) guidelines for respiratory infectious diseases: A rapid qualitative evidence synthesis. Cochrane Database Syst Rev 2020;4:14651858.CD013582:1-55.

79 Fatima R, Akhtar N, Yaqoob A. Building better tuberculosis control systems in a postCOVID world: learning from Pakistan during the COVID-19 pandemic. Int I Infect Dis 2021;24:j.jijid.2021.03.026.

80 Stop TB Partnership. The paradigm shift: global plan to end TB 2018-22, 2019. 